\title{
Tendances et concepts nouveaux en matière de protection contre les radiations
}

\author{
G. DARDENNE (*) \\ (Manuscrit reçu le 14 mars 1978)
}

RÉSUMÉ

Les fondements de la notion d'homme-rem et de celle de dose collective qui lui correspond sont examinés. Les formules exprimant la dose collective ou le débit de dose collectif sont justifiées et un certain nombre d'applications pratiques sont indiquées.

\begin{abstract}
The foundations of the notion of "man-rem" and of the corresponding collective dose are examined. Formulae expressing collective dose and collective dose rate are justified. Some practical applications are pointed out.
\end{abstract}

\section{NOTION DE DOSE COLLECTIVE. JUSTIFICATION}

Jusque vers 1955, le risque d'irradiation concernait essentiellement un petit nombre de personnes travaillant sur des appareils bien localisés : générateurs de rayons $X$, sources de radium ou cobalt - utilisés pour le diagnostic ou le traitement - accélérateurs de particules... L'évaluation du risque éventueI était alors effectuée au niveau individuel : par exemple, pour l'individu irradié, une dose de $D$ rad d'un rayonnement donné entraîne un risque supplémentaire d'être atteint de leucémie dans le cours de son existence. Sur cette base, des limites individuelles d'équivalents de doses admissibles avaient été établies $\left(^{1}\right)$.

(*) IN2 P3, Laboratoire de l'accélérateur linéaire, Centre d'Orsay, Bât. 200, 91405 Orsay.

( $\left.{ }^{1}\right) 5 \mathrm{rem} / \mathrm{an}$ pour les travailleurs professionnellement exposés; $0,5 \mathrm{rem} / \mathrm{an}$ pour les personnes du public.

RADIOPROTECTION, VOL. 13 - $\mathrm{N}^{\circ} 4$ 
Disons, pour fixer les idées, que ceci correspondait à un risque de l'ordre de $10^{-5}$ ou $10^{-6}$, considéré comme négligeable par chaque individu dans les sociétés évoluées.

Depuis une quinzaine d'années, deux ordres de faits ont conduit à considérer l'éventualité d'irradiations de populations nombreuses, à la fois dans l'espace et dans le temps :

- le développement des centrales nucléaires;

- le développement des armes nucléaires;

L'analyse risque-bénéfice s'est alors transposée d'un niveau individuel au niveau collectif, la population $P$ étant considérée comme une entité pour laquelle on peut admettre tel dommage - par exemple tel nombre de leucémies par an - compte tenu du «bénéfice » que lui apporte, par ailleurs, l'énergie nucléaire. Mais le risque collectif ne peut être évalué que si l'on dispose d'une relation entre la dose distribuée à la population $P$ et le dommage qui en résulte pour cette population.

Considérons donc un dommage donné, par exemple le décès par suite de leucémie, et une population $P$ pendant une période donnée, disons une génération de $N$ individus entre 25 et 60 ans. Parmi ces $N$ individus, supposons que $n$ aient reçu chacun une dose $D$ identique, uniformément distribuée au cours de la période considérée et soit $L$ le nombre d'apparitions de dommages dans $P$.

Gardons $D=\mathrm{C}^{\text {te }}$ et faisons varier $n$. Le nombre de dommages augmente évidemment de façon proportionnelle au volume de la population irradiée. On a une fonction $L=f_{1}(n)$ qui est croissante et linéaire : $L=\alpha n$.

Gardons $n=C^{\text {te }}$ et faisons varier $D$; on a une fonction $L=f_{2}(D)$. Deux considérations conduisent à admettre pour cette fonction aussi une forme linéaire croissante à partir de $D=0$ :

- d'une part, les résultats expérimentaux obtenus dans la région des fortes doses $(D>20 \mathrm{rad})$;

- d'autre part, en ce qui concerne les effets des faibles doses et des faibles débits de dose, le souci de retenir les hypothèses qui donnent le maximum de garanties pour la santé des populations. En effet, dans la région des faibles doses et débits, l'expérimentation ne donne pas de résultats significatifs, du fait des fluctuations statistiques et de l'impossibilité d'irradier des populations suffisamment nombreuses. On en est donc réduit aux hypothèses et les plus conservatrices donnent : $L=\beta D$. Au total, $L$ étant proportionnel à $n$ et à $D$ est proportionnel au produit $x=n . D$, lequel s'exprime en homme-rem. On a $L=K x$ et à une valeur donnée de $x$ correspond une valeur de $L$ et une seule. Par exemple, $x=5.10^{5}$ si $10^{5}$ individus sont irradiés à 5 rem mais aussi bien si $10^{6}$ individus sont irradiés à 0,5 rem; le nombre d'apparitions de dommages sera donc le même dans les deux cas. La diminution du risque avec la dose au niveau individuel est compensée par l'augmentation du risque au niveau collectif du fait de l'élargissement de la population irradiée.

L'évaluation du risque pour une population?est ainsi ramenée à celle de la dose collective. 


\section{FORMULATION MATHÉMATIQUE}

DOSE COLLECTIVE DANS LE CAS D'UNE IRRADIATION INSTANTANÉE (par exemple : irradiations médicales, explosions nucléaires...)

Soit une population $P$ de $N$ individus. $P$ étant large, ces individus auront, en général, reçu des doses différentes.

D'après ce qui précède, il faut alors partager $P$ en sous-groupes $p_{1}, p_{2}, \ldots$ d'irradiation homogène :

- les $\Delta n_{1}$ individus de $p_{1}$ ont reçu chacun $D_{1}$ rem;

- les $\Delta n_{2}$ individus de $p_{2}$ ont reçu chacun $D_{2}$ rem;

- etc.

La dose collective sera donnée par :

$$
D_{c}=D_{1} \cdot \Delta n_{1}+D_{2} \cdot \Delta n_{2}+\ldots
$$

De façon générale, on envisagera une fonction $n=f(D)$ continue et dérivable et les expressions suivantes :

$(d n / d D)(D)$, taux de variation au voisinage de $D$ de la population irradiée, en hommes par rem;

$(d n / d D)(D) . d D$, petite fraction de la population dont les individus ont reçu des doses comprises entre $D$ et $D+d D$;

$D \cdot d n / d D(D) d D$, dose collective pour la fraction de population en question, en homme-rem.

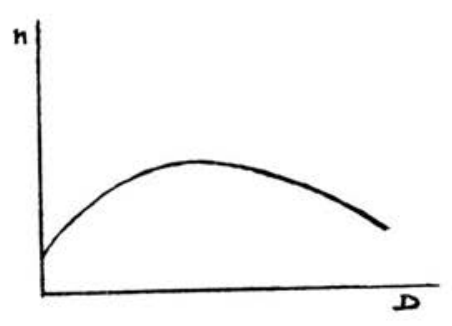

La dose collective totale sera la somme des doses différentielles :

$$
D_{c}=\int_{0}^{\infty} D \cdot \frac{d n}{d D}(D) d D \text { homme-rem. }
$$

Dose COllective Dans Le CAS D'UNE IRRADIATION VARIABle PENDANT UNE PÉRIODE T.

Dans le cas des retombées radioactives d'une explosion nucléaire ou des rejets radioactifs des centrales, les irradiations se prolongent pendant des années ou des dizaines d'années [parmi les isotopes à longue période rejetés dans l'atmosphère, citons ${ }^{137} \mathrm{Cs}$ (33 ans), ${ }^{90} \mathrm{Sr}(25$ ans), ${ }^{129} \mathrm{I}\left(1,7 \cdot 10^{7}\right.$ ans $),{ }^{238} \mathrm{U}\left(4,5 \cdot 10^{9}\right.$ ans $\left.)\right]$. La dose individuelle $D$ reçue est alors fonction de la durée de l'irradiation : $D=f(t)$ et il faut considérer le débit de dose instan-

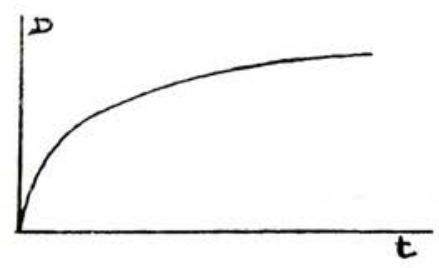


tané $J$, qui radioactifs va en diminuant :

$$
J=\frac{d D^{1}}{d t}(t)
$$

On définit alors un débit de dose collectif $J_{c}$ à l'instant $t$ par

$$
J_{c}=\frac{d D_{c}}{1 d t}(t)
$$

ce qui revient à dériver sous le signe $\int 1$ 'expression (2) :

$$
J_{c}(t)=\int_{0}^{\infty} \frac{d D^{1}}{d t}(t) \cdot \frac{d n}{d D}(D) d D \text { homme } \frac{\text { rem }}{s}
$$

Sous une autre forme, on peut considérer que l'on pondère le débit de dose par le nombre d'individus qui ont été soumis à ce débit.

$J_{c}$ est évidemment aussi une fonction de $t$ et on obtient la dose collective distribuée à la population pendant la période $T$ en intégrant $J_{c}$ sur le temps

$$
D_{c}=\int_{0}^{T} J_{c}(t) d t \text { homme-rem. }
$$

\section{APPLICATIONS PRATIQUES}

La valeur de (3) - et donc celle de (4) - reste inchangée quand on élargit arbitrairement la population $P$ en y incluant des individus non irradiés $(d D / d t=0)$. Il est intéressant dès lors de considérer l'ensemble de la population mondiale.

Dose COlLeCtive due a L'irRadiation NATURELle. BASE DE RÉfÉRENCE.

Admettons que la dose individuelle annuelle due aux radiations du sol, cosmiques et du corps humain lui-même soit de l'ordre de $10^{-1}$ rem en moyenne et que la population du globe soit de $3.10^{9}$ habitants. La dose collective/naturelle est $D_{c n}=3 \cdot 10^{8}$ homme-rem/an.

IRRADIATIONS MÉDICALES POUR DIAGNOSTIC

Admettons en moyenne par individu et par diagnostic : $10^{-1} \mathrm{rem}$. Si on estime qu'un tiers de la population mondiale subit un radiodiagnostic par an, on a : $D_{c m}=10^{8}$ homme-rem/an.

( $\left.{ }^{1}\right)$ Les chiffres donnés ci-après sont extraits de la communication de D. Beninson [1]. 


\section{EXPLOSIONS NUCLÉAIRES}

La dose collective, au niveau des gonades, intégrée sur le temps, due aux explosions nucléaires dans l'atmosphère effectuées avant 1976 serait de l'ordre de $D_{c e}=3,6.10^{8}$ homme-rem. Ceci correspond à 14 mois d'exposition aux radiations naturelles.

\section{VOYAGES EN AVION}

Chaque année, les voyages aériens correspondent à $10^{9}$ heure.passager environ. Le débit de dose moyen en altitude de croisière étant de $3.10^{-4} \mathrm{rem} / \mathrm{h}$, on a une dose collective annuelle de l'ordre de $3.10^{5}$ homme-rem.

\section{Centrales NUClÉAires}

Une année de fonctionnement de toutes les centrales nucléaires opérationnelles en 1976 donne une dose collective intégrée sur le temps de l'ordre de $5.10^{5}$ homme-rem soit l'équivalent de $\left(5.10^{5} / 3.10^{8}\right) \times 365=0,6$ journée d'irradiation naturelle.

Les centrales envisagées pour l'an 2000 donneraient $\simeq 10^{7}$ homme-rem. On voit que les centrales nucléaires se situent loin derrière les autres sources d'irradiation de la population mondiale - si l'on met à part les voyages en avion.

\section{CONSÉQUENCES EN CE QUI CONCERNE LES LIMITES MAXIMALES ADMISSIBLES INDIVIDUELLES}

Les notions qui précèdent sont largement utilisées, notamment pour l'étude, la construction et le contrôle du fonctionnement des centrales nucléaires.

Elles ont, par ailleurs, conduit à une interprétation sensiblement plus restrictive des normes individuelles - limites maximales admissibles (LMA). De nouvelles recommandations ont été publiées récemment à ce sujet par la Commission Internationale de Protection Radiologique (CIPR) [3].

a) Les anciennes LMA sont maintenues inchangées :

- 5 rem/an.pour les personnes directement exposées à des travaux sous radiations,

$-0,5 \mathrm{rem} / \mathrm{an}$ pour les personnes

Mais ces doses ne doivent pas être considérées comme des rations auxquelles chaque individu aurait droit. La CIPR a tenu compte du fait que, en pratique au cours des 20 dernières années, la grande majorité des doses reçues se situait très largement au-dessous des LMA et elle a clairement indiqué que si tel n'avait pas été le cas elle se serait prononcée pour une diminution des LMA ci-dessus indiquées. D'où un second principe :

b) Les LMA sont des doses admissibles dans des cas isolés, mais il faut toujours faire en sorte que, pour la majorité des personnes, les irradiations se 
situent le plus bas possible au-dessous des LMA. Autrement dit, il ne serait pas admissible de délivrer 5 rem par an à tous les travailleurs des industries nucléaires et les consignes suivantes doivent être respectées :

- toute irradiation de personnel doit être justifiée : il faut un " enjeu » suffisant et une grosse difficulté pratique ou économique d'effectuer le travail sans irradiation;

- toute irradiation doit être optimisée. Les facteurs temps, distance, écrans doivent être combinés de façon à obtenir des doses suffisamment basses au-dessous de la LMA.

\section{BIBLIOGRAPHIE}

[1] BEnINSON D. Collective doses from practices involving radiation exposures. IRPA., $I V^{\circ}$ Congrès International, Paris, 24-30, avril 1977, 3, 693-899.

[2] Kahan R. S., Feige Y. The wide margin of implicit built-in safety factors in current radiation protection standards. Health Phys., 1977, 32, 509-521.

[3] I.C.R.P. Recommendations of the International Commission on Radiological Protection, ICRP publication 26. Oxford, Pergamon press, 1977.

N.D.L.R. L'article précédent de M. Dardenne présente la position officielle de la Commission Internationale de Protection Radiologique, mais l'on doit noter que celle-ci est sujette à de nombreuses critiques (voir, en particulier, A. M. Weinberg : Benefit/cost analysis and the linear hypothesis, dans Nature, vol. 271 du 16 février 1978).

En effet, si l'idée de l'analyse risque/bénéfice est séduisante, elle est difficile à mettre en œuvre car les mesures du risque (santé) et du bénéfice (énergie) ne sont pas les mêmes. Dans le cas présent, cette analyse est basée d'une part sur l'utilisation de l'homme-remcomme estimateur de dommage et sur l'hypothèse non justifiée («probablement invérifiable et sans doute fausse" ", dit Weinberg) de linéarité des effets biologiques en fonction de la dose (y compris pour les très faibles doses).

Il faut donc utiliser avec prudence les conclusions des études de risque/bénéfice faites sur ces bases. 